\title{
A study on performance of two serological assays for diagnosis of leprosy patients
}

\author{
OM PARKASH, V. CHATURVEDI*, \\ B. K. GIRDHAR \& U. SENGUPTA \\ Central JALM A Institute for Leprosy, Tajganj, Agra-282001, India
}

Accepted for publication 1 July 1994

Summary We compared 2 serological tests for the diagnosis of leprosy to test their performances. The tests include the serum antibody competition test (SACT) for the detection of antibodies to Mycobacterium leprae-specific epitope on $35 \mathrm{KDa}$ protein molecule, and $M$. leprae gelatin particle agglutination assay (MLPA), for the detection of antiphenolic glycolipid-1 (PGL-1) antibodies. In both the assays a higher serological positivity was seen amongst multibacillary (MB) patients than those in paucibacillary (PB) patients. Taking all leprosy patients together, the sensitivity of SACT $(59 \cdot 7 \%)$ was observed to be statistically comparable to that of MLPA (66.9\%). However, SACT proved to be more specific (97.7\%) than MLPA (75.0\%). The agreement between these 2 assays was observed to be moderate.

\section{Introduction}

Leprosy is a major world health problem and in order to eliminate this disease two main strategies, accurate diagnosis and treatment, are playing a pivotal role. Recently a wide variety of serological tests have been developed for diagnosing leprosy patients by detecting anti $M$. leprae antibodies in their sera. ${ }^{1}$ Of the assays described so far, the phenolic glycolipid-1 (PGL-1) utilizing enzyme-linked immunosorbent assay (PGLELISA), and the serum antibody competition test (SACT), have been studied widely and have been suggested to be highly promising for the serodiagnosis and monitoring of chemotherapy in lepromatous leprosy patients. ${ }^{2-7}$ Recently, a microagglutination assay, called MLPA, that detects antiPGL-1 antibodies, has been introduced by Izumi et al. ${ }^{8}$ It is a rapid and simple assay which can be evaluated by the naked eye. Further, its sensitivity and specificity have been shown to be comparable to that of PGL-ELISA. The present study was designed to evaluate the performances, in terms of sensitivities and specificities for diagnosis of leprosy patients, of SACT and MLPA using a set of sera. India.

${ }^{*}$ Correspondence: Department of Microbiology, Central Drug Research Institute (CDRI), Lucknow, 


\title{
Materials and methods
}

\author{
SERUM SAMPLES
}

Sera were obtained from 68 multibacillary (MB) and 56 paucibacillary (PB) leprosy patients (who were active cases receiving antileprosy treatment) attending the hospital at the Central JALMA Institute for Leprosy, Agra, India. Sera from 28 pulmonary tuberculosis patients and 16 healthy volunteers, working at the Institute laboratories, were also included as controls. The sera were stored at $-20^{\circ} \mathrm{C}$ until use.

\section{DETECTION OF $M$. LEPRAE SPECIFIC ANTIBODIES USING SACT}

In this approach, antibodies against a $M$. leprae specific epitope on $35 \mathrm{KDa}$ antigen of $M$. leprae were detected by the competition test. ${ }^{5,7}$ Briefly, the wells were coated with $M$. leprae antigen (supplied by IMMLEP, WHO, through Dr R. J. W. Rees). The wells were then incubated with peroxidase conjugated $M$. leprae specific monoclonal antibody (MLO-4) in the presence or absence of a 10 -fold dilution of serum. Finally, colour was developed using o-phenylene-diamine dihydrochloride as substrate and optical density values were then read at $492 \mathrm{~nm}$ using an ELISA reader. Serum causing $50 \%$ or more inhibition of the binding of enzyme conjugated antibody to the specific epitope was regarded as positive.

\section{DETECTION OF ANTIPGL-1 ANTIBODIES USING MLPA KiT}

The microtitre agglutination test for screening of leprosy patients was performed using the Serodia leprae diagnostic kit (manufactured by Fusirebio Inc., Tokyo, Japan). Briefly, serum samples were diluted to $1: 8$ and $1: 16$ in wells of U-bottom microtitre plates giving a final volume of $25 \mu \mathrm{l} /$ well. Then equal amounts of unsensitized and antigen-sensitized gelatin particles were mixed with these diluted sera giving $1: 16$ and $1: 32$ as the final dilutions. The contents in the wells were mixed thoroughly. Plates were covered and incubated at room temperature for $2 \mathrm{hr}$. Upon completion of the reaction the agglutination in the wells were read. Serum samples showing agglutination at $1: 32$ were taken as positive.

\section{Results and discussion}

Table 1 shows the positivities achieved by the 2 assays in various groups. In common with previously reported findings with SACT and MLPA ${ }^{2,5,8}$ we have also found high sensitivity in the MB group of patients compared with the PB group of patients. When considering MB patients, the highest positivity ( 66 out of 68 ) was observed using SACT. MLPA showed more positivity in the case of the PB group of patients (21 out of 56). Taking all the patients together, the MLPA positivity was slightly more than SACT (83 vs 74). With MLPA, 4 out of 16 and 7 out of 28 sera were found to be positive in healthy individuals and tuberculosis patients, respectively. On the other hand, there was less false positivity with SACT, whereas all of the 16 sera from healthy subjects and 27 from 28 tuberculosis patients were negative. In all, only 1 out of 44 sera from nonleprosy 
Table 1. Positivities for anti- $M$. leprae antibodies in sera from various groups using SACT and MLPA tests

\begin{tabular}{lccc}
\hline Subjects & $\begin{array}{c}\text { Number of } \\
\text { sera tested }\end{array}$ & $\begin{array}{c}\text { SACT } \\
\text { positive }\end{array}$ & $\begin{array}{c}\text { MLPA } \\
\text { positive }\end{array}$ \\
\hline Controls & & & \\
$\quad$ Healthy & 16 & 0 & 4 \\
$\quad$ Tuberculosis & 28 & 1 & 7 \\
Total & 44 & 1 & 11 \\
Leprosy patients & & 66 & 62 \\
$\quad$ Multibacillary & 68 & 8 & 21 \\
Paucibacillary & 56 & 74 & 83 \\
Total & 124 & & \\
\hline
\end{tabular}

controls (healthy persons and tuberculosis patients) was positive for SACT, whereas 11 of these 44 sera were positive using the MLPA test.

Based upon the above data, the percent sensitivities and specificities for the detection of leprosy patients of the 2 assays were determined. Table 2 shows these values. The results indicate that the sensitivity of SACT was $59.7 \%$, whereas that of MLPA was $66 \cdot 9 \%$. The specificity of SACT was shown to be $97 \cdot 7 \%$ and that of MLPA was $75 \cdot 0 \%$.

On analysis, though the sensitivity of MLPA was found to be slightly more than SACT, statistically there was no difference between the 2 tests $\left(\chi^{2}=1 \cdot 116 ; p<0 \cdot 1\right)$. As far as we know, the only report comparing MLPA and SACT which described MLPA as more sensitive than SACT is that of Dhandaya Chapani et al. ${ }^{9}$ Our findings contradict their report. The reason for this discrepancy could be that in the previous study, analysis for sensitivity was carried out taking both leprosy patients as well as nonleprosy individuals into consideration, while in the present study we included leprosy patients only for this purpose.

Further, we have found (Table 2) that SACT is significantly more specific $\left(\chi^{2}=21.859 ; p<0.001\right)$ than MLPA. In order to distinguish between nonspecific agglutination reaction and agglutination due to antiPGL antibodies, the antiPGL-1ELISA was run in parallel with MLPA. The results have shown that of the 11

Table 2. Sensitivities and specificities of SACT and MLPA for detection of anti$M$. leprae antibodies in leprosy sera

\begin{tabular}{lcc}
\hline Test & $\begin{array}{c}\text { Sensitivity } \\
(\%)\end{array}$ & $\begin{array}{c}\text { Specificity } \\
(\%)\end{array}$ \\
\hline SACT & 59.7 & 97.7 \\
MLPA & 66.9 & 75.0 \\
\hline
\end{tabular}

Sensitivity of SACT and MLPA, for detection of leprosy patients, did not differ significantly $(p<0 \cdot 1)$.

SACT was more $(p<0.001)$ specific than MLPA. 
Table 3. Agreement between SACT and MLPA tests for the detection of antiM. leprae antibodies using 124 leprosy sera

\begin{tabular}{lcc}
\hline & \multicolumn{2}{c}{ MLPA results } \\
\cline { 2 - 3 } SACT results & Positive & Negative \\
\hline Positive & 64 & 10 \\
Negative & 19 & 31 \\
\hline
\end{tabular}

Percent agreement, $76 \cdot 7 \% ; \kappa$ value, $0 \cdot 5$.

nonleprosy controls (which were positive by MLPA) only 2 were positive by PGL-1 ELISA (detailed data not given) indicating that the nonspecific positivities in MLPA were probably due to false agglutination caused by biophysical properties of sera from certain individuals, as has been proposed by Chanteau et al. than found in this work. ${ }^{10}$ In contrast to our findings, Izumi et al. ${ }^{8}$ demonstrated a lower frequency of MLPA positive sera among normal healthy persons and tuberculosis patients. These contradictory findings might be due to individuals belonging to different ethnic groups and locations that were used in these 2 studies.

As shown in Table 3 agreement $(76 \cdot 7 \%)$ between the 2 tests was found to be moderate (indicated by a $\kappa$ value of $0 \cdot 5$ ). Some disagreement was expected, because the 2 assays measure different antibodies.

In conclusion, the sensitivity of SACT appears comparable to that of MLPA and SACT is more specific than MLPA. However, neither of these 2 assays seems to be very promising, even in diagnosing established leprosy. Therefore there is need of more specific and sensitive test(s) for the diagnosis of leprosy patients for use in screening the population.

\section{Acknowledgments}

We thank Dr S. Izumi, National Leprosy Research Institute, Tokyo, Japan; Dr R. J. W. Rees, IMMLEP Bank, National Institute for Medical Research, London, and Dr Juraj Ivanyi, Tuberculosis and Related Diseases Research Unit, Hammersmith Hospital, London, for a free supply of MLPA kits, $M$. leprae antigen and monoclonal antibodies (MLO-4), respectively. We are grateful to LEPRA, UK, for donating some of the reagents used in this study. Our thanks are also due to Mr Malikhan Singh, Mr P. N. Sharma, Mr M. Alam for their technical assistance and to Mr A. S. Bhatia and V. S. Yadav for their suggestions regarding statistical analysis.

\section{References}

1 Anonymous. Serological tests for leprosy. Lancet, 1986; 8480: 533-5.

2 Young DB, Buchannan TM. A serological test for leprosy with a glycolipid specific for M. leprae. Science, 1983; 221: 1057-9. 
${ }^{3}$ Cho SN, Fujiwara T, Hunter SW, Rea TH, Gelbar RH, Brennan PJ. Use of an artificial antigen containing the 3,6-di-o-methyl-B-D-glucopyranosul epitope for serodiagnosis of leprosy. J Infect Dis, 1984: 150: 31122.

4 Douglas JT, Steven LM, Fajarado T, Cellona RV, Madarang MG, Abolas RM, Steenbergen GJ. The effect of chemotherapy on antibody levels in lepromatous patients. Lepr Rev, 1988; 59: 127-35.

5 Sinha S, Sengupta U, Ramu G, Ivanyi J. A serological test for leprosy based on competitive inhibition of monoclonal antibody binding to the MY2a determinant of Mycobacterium leprae. Trans $R$ Soc Med Hyg, 1983; 77: 869-71.

${ }^{6}$ Sinha S, Sengupta U, Ramu G, Ivanyi J. Serological survey of leprosy and control subjects by a monoclonal antibody based immunoassay. Int J Lepr, 1985; 53: 33-38.

7 Sinha SK, McEntegart A, Girdhar BK, Bhatia AS, Sengupta U. Appraisal of two M. leprae specific serological assays for chemotherapy in lepromatous (LL/BL) leprosy patients. Int J Le pr, 57: 24-32.

8 Izumi S, Fuziwara T, Ikeda M, Nishimura Y, Sugiyama K, Kawats K. Novel gelatin particle agglutination test for serodiagnosis of leprosy in the field. J Clin Microbiol, 1990; 28: 525-9.

9 Dhandayuthapani S, Anandan D, Bhatia VN. Evaluation of MLPA test for serodiagnosis of leprosy. Int J Lepr, 1992; 60: 84-6.

10 Chanteau S, Cartel JL, Boutin JP, Roux J. Evaluation of gelatin particle agglutination assay for the detection of antiPGL-1 antibodies. Comparison with ELISA method and applicability on a large scale study using blood collection on filter paper. Lepr Rev, 1991; 62: 255-61. 